\title{
DEGRADACIÓN DE HIDROCARBUROS AROMÁTICOS POLICÍCLICOS DE RESIDUOS DE BARRIDO DE CALLES USANDO COMPOST
}

\author{
Lizardo Visitación Figueroa*a, Freddy J. Rojas ${ }^{\mathrm{b}}$, Víctor Meza Contreras ${ }^{\mathrm{a}}$, \\ Lisveth Flores del Pino ${ }^{\mathrm{a}}$
}

\begin{abstract}
RESUMEN
En el presente estudio se evaluó la degradación de Hidrocarburos Aromáticos Policíclicos (HAPs) provenientes de residuos de barrido de calles utilizando tratamiento aeróbico con dos tipos de compost a diferente grado de maduración. La degradación fue evaluada en mezclas al 75\% de residuo de barrido de calles con compost a intervalos de 0, 28, 60, 90 y 120 días. Se logró una mayor remoción al $80 \%$ y $90 \%$ de la sumatoria de HAPs ( 2 HAPs) en 120 días de tratamiento en compost sin y con pretratamiento anaeróbico, respectivamente; esto posiblemente influenciado por la presencia de microorganismos con capacidad de degradar materia orgánica y producir sustancias húmicas. El efecto tóxico evaluado como BaPeq se reduce en más del $66 \%$ en los 30 primeros días para las muestras con tratamiento y más de 90 días para la muestra control. El efecto ecotoxicológico evaluado sobre Daphnia magna y Lactuca sativa, nos indican que los HAPs al ser cometabolizados producen metabolitos con mayor efecto ecotoxicológico, principalmente entre los 30 y 60 días, reduciendo su efecto a más de 90 días de tratamiento.
\end{abstract}

Palabras clave: HAPs, compost, sustancias húmicas, residuos de barrido de calles.

\section{POLYCYCLIC AROMATIC HYDROCARBON DEGRADATION FROM WASTE STREET SWEEP BY COMPOST}

\begin{abstract}
In this study the degradation of polycyclic aromatic hydrocarbons (PAHs) present in waste street sweeping by aerobic treatment was assessed using four different samples of compost quality. It was possible to reduce the concentration in the samples $\Sigma$ HAPs reaching removal rates of greater than $80 \%$ within 120 days of aerobic treatment, samples of compost with anaerobic pretreatment promote further degradation of PAHs reaching higher removals than $90 \%$, this may influenced by the presence of microorganisms capable of degrading organic matter and humic substances produce. The toxic effect evaluated as BaPeq is reduced by

\footnotetext{
${ }^{a}$ Centro de Investigación en Química, Toxicología y Biotecnología Ambiental del Departamento Académico de Química de la Facultad de Ciencias de la Universidad Nacional Agraria La Molina. Lima - Perú, lvisitacion@ lamolina.edu.pe

b Pontificia Universidad Católica del Perú, Ingeniería Mecánica - Área Energía, Av. Universitaria 1801, San Miguel (Lima-Perú), Tel: (511) 6262000 (Anexo 4820).
} 
over $66 \%$ in the first 30 days of treatment for waste shows similar percentages sweep streets can be achieved in more than 90 days of treatment. The ecotoxicological effects assessed on Daphnia magna and Lactuca sativa, indicate that PAH metabolites being produced cometabolizados more ecotoxicological effect mainly between 30 and 60 days, reducing its effect over 90 days of treatment.

Key words: PAHs, compost, humic acids residues streets.

\section{INTRODUCCIÓN}

Los residuos de barrido de calles (RBC) tienen una alta ecotoxicidad y toxicidad, por la presencia de varias sustancias contaminantes, tales como los HAPs, estos residuos pueden ser tratados utilizando mezclas de compost, debido a que este material tiene una gran capacidad para sostener poblaciones de microorganismos capaces de degradar una variedad de contaminantes orgánicos como los HAPs mineralizándolos hasta $\mathrm{CO}_{2}$ o fijándolos irreversiblemente en la matriz sólida ${ }^{1,2,3}$.

La influencia de la adición de compost sobre la degradación de los HAPs en RBC y suelos puede deberse a tres mecanismos: la adsorción de los HAPs sobre la matriz sólida, el compost como fuente de nutrientes, microorganismos asociados a la matriz orgánica de la mezcla del compost y del residuo. Estudios realizados sobre estos mecanismos han determinado que la presencia de los microorganismos de la matriz compost, residuos o suelo es el principal aporte del compost, existiendo una fase previa de adaptación2, donde el cometabolismo es el mecanismo frecuentemente utilizado para la biodegradación de los HAPs ${ }^{3}$.

Los microorganismos capaces de degradar sustancias húmicas presentes en muestras de compost maduro tienen potencial para degradar los HAPs presentes en suelos o residuos sólidos ${ }^{3}$, además la adición de compost maduro mejora significativamente la actividad microbiana del suelo, las condiciones del suelo, la disponibilidad de nutrientes, facilitando la degradación de los HAPs ${ }^{4}$.

Compost producido por la mezcla de rastrojo vegetal (paja, hojas, recortes o tallos) como fuente de carbono y excretas animales (oveja, pollo, vacunos) han probado capacidad para biodegradar HAPs y otros contaminantes orgánicos ${ }^{3,5}$.

En el Perú son limitados los trabajos realizados sobre HAPs en muestras de residuos y sus efectos, motivo por el cual nuestro objetivo para la presente investigación fue evaluar la degradación de los HAPs en RBC utilizando dos tipos de compost en dos grados de maduración, mediante la medición de la concentración individual de cada HAP, $\Sigma$ HAPs, determinación del BaPeq donde se incluye el efecto carcinogénico de los 16 HAPs, y test ecotoxicológicos. 


\title{
PARTE EXPERIMENTAL
}

\section{Toma de muestra}

Las muestras de RBC fueron tomadas de la Av. Abancay, en el Centro de Lima, durante los meses de octubre y noviembre del 2014 , la recolección fue realizada de forma manual, con un suave barrido a lo largo de toda la avenida ${ }^{6}$, una hora previa al paso del personal de limpieza (23:00 horas). Las muestras fueron secadas y almacenadas a $4{ }^{\circ} \mathrm{C}$, previo a los ensayos de degradación, las muestras de los diferentes días fueron homogenizadas.

\section{Caracterización fisicoquímica de las muestras de compost}

Se trabajó con dos tipos de compost en dos grados de maduración: 1) compost producido por la Asociación XRunner, elaborado de aserrín y excretas, inmaduro (XI) de 6 meses y maduro (XM) de 1 año, estas muestras de compost han tenido un pretratamiento anaerobio de 20 a 30 días. 2) Compost producido por el Centro Modelo de Tratamiento de Residuos (CEMTRAR) de la UNALM elaborado de rastrojo vegetal y excretas, inmaduro (CI) de 2 meses y maduro (CM) de 4 meses, sin pretratamiento anaerobio inicial.

Los parámetros medidos para caracterizar las muestras de compost fueron el porcentaje de humedad, ceniza, perdida por ignición (PPI) y la relación carbono nitrógeno $\mathrm{C} / \mathrm{N}^{7}$. La madurez del compost fue evaluada mediante el porcentaje de ácidos húmicos ${ }^{8}$.

\section{Degradación de HAPs en muestras de compost}

Los RBC fueron mezclados con las muestras de compost al $75 \%$, se colocó $5 \mathrm{~kg}$ de las mezclas en un reactor tubular de $12 \mathrm{~L} \mathrm{a} 35^{\circ} \mathrm{C}$, las muestras fueron aireadas 2 veces a la semana por rotación manual. Se tomaron submuestras de $5 \mathrm{~g}$ del proceso de degradación a los $0,28,60,90$ y 120 días, con la finalidad de medir la concentración residual de HAPs ${ }^{9}$. Las concentraciones de los diferentes HAPs, en función del tiempo, fueron evaluados utilizando una reacción de seudo primer orden. Se estimó el tiempo de vida media de cada HAP en los $\mathrm{RBC}$ con y sin tratamiento con compost ${ }^{10}$.

\begin{abstract}
Análisis de HAPs
La extracción de HAPs, de las mezclas de RBC con compost, fue realizada a partir de $5 \mathrm{~g}$ de muestra en el equipo Ultrasonic Cleaner Modelo UC-20 de la marca Jeio Tech con una frecuencia ultrasónica de $40 \mathrm{KHz}$ a escala media y $25^{\circ} \mathrm{C}$ de temperatura ${ }^{11}$, por un intervalo de 10 minutos, utilizando una mezcla al 10\% de éter en hexano y 2 g de sulfato de sodio anhidro ${ }^{12}$. Se purificó el extracto con una columna de $0,75 \mathrm{~cm}$ de radio con $2 \mathrm{~g}$ de silicagel 60 (30-70 mesh). Para la determinación de los HAPs se utilizó el Sistema de Cromatografía de Gases Agilent Technologies 7890A, con detector de masas $5975 \mathrm{C}$ y automuestreador modelo 120, columna Zebron ZB-5MS Capilar de 30m x 0,32mm x 1,00um, gas de corrida Helio $0,6 \mathrm{~mL} / \mathrm{min}$ con flujo constante.
\end{abstract}

\section{Efecto ecotoxicológico y toxicológico}

Para la obtención del extracto de lixiviación de las submuestras tratadas se empleó el Procedimiento de Caracterización de un lixiviado por su toxicidad "Toxicity Characteristic 
Leaching Procedure" (TLCP) utilizando una dilución del residuo 20:1 con un extractante a $\mathrm{pH}=4,9^{13,14}$.

Se evaluó la ecotoxicidad de las submuestras siguiendo los lineamientos del protocolo de la EPA 600/3-88/029 para test agudos de lugares con residuos peligrosos evaluados sobre el lixiviado ${ }^{15}$. Se utilizó como organismos de prueba neonatos de Daphnia magna con 24 horas de nacidos, evaluando la concentración que ocasiona un efecto tóxico al $50 \%$ de la población (EC50) durante 24 horas $^{16-18}$, semillas de Lactuca sativa evaluando el índice de germinación $(\operatorname{Ig})^{17}$.

Los parámetros toxicológicos fueron transformados en unidades tóxicas (UT), mediante el uso de la fórmula de $U T=\frac{c}{C E_{x}}$ donde $\mathrm{C}$ es la concentración del residuo máxima utilizada (en este caso $100 \%$ ) y CEx es la concentración que ocasiona efecto, en el trabajo se utilizó CE50 efecto al $50 \%$ de la población ${ }^{18-20}$.

La toxicidad de las muestras tratadas y sin tratar fue determinado utilizando los Factores de Equivalencia Tóxica (FET) propuestos por Nisbet y LaGoy, siendo el BaP la sustancia de referencia más tóxica ${ }^{21}$. La Concentración Tóxica Equivalente (CTE) de la muestras RBC se determinó por la suma de los productos de la concentración de cada HAP individual determinado en un estudio anterior y su FET ${ }^{11,22}$.

\section{Correlación entre calidad del compost y degradación de los HAPs}

Se elaboró una matriz de correlación de Pearson entre los parámetros de calidad del compost y la sumatoria de la concentración de los HAPs y BaP equivalente al final de los tratamientos, con la finalidad de observar la influencia de la calidad del compost en la eficiencia de degradación de los HAPs.

\section{RESULTADOS Y DISCUSIÓN}

\section{Caracterización Fisicoquímica del Compost}

Como se observa en la tabla 1, las muestras provenientes de CEMTRAR presentan menores valores de humedad, material orgánico como pérdida por ignición PPI, relación $\mathrm{C} / \mathrm{N}$, mayores valores de ceniza y porcentaje de ácidos húmicos (\% HA) que las muestras de XRUNNER. Las muestras de CEMTRAR difieren significativamente de las muestras de XRUNNER.

Tabla 1. Resultados de caracterización del compost

\begin{tabular}{lccccc}
\hline & \% Humedad & Ceniza & PPI & C/N & \% HA \\
\hline CM & 34,15 & 41,5 & 35,08 & 11,97 & 9,9 \\
CI & 18,81 & 43,9 & 43,61 & 12,63 & 8,9 \\
XI & 80,44 & 3,9 & 79,62 & 14,42 & 4,0 \\
XM & 54,42 & 23,3 & 48,43 & 14,8 & 1,9 \\
\hline
\end{tabular}


La producción del compost de XRUNNER, al obtenerse por varios procesos con bajo contenido de oxígeno, presenta contenidos de ácidos húmicos menores al de CEMTRAR, donde se obtiene íntegramente por procesos aeróbicos, situación similar ha sido descrita por Binner et al., 2007.

\section{Degradación de HAPs en muestras de compost}

El contenido inicial de HAPs en RBC (tabla 2) expresado como $\Sigma$ HAPs presenta un valor de $2,7234 \mu \mathrm{g} / \mathrm{g}$ y como BaPeq de $0,2824 \mu \mathrm{g} / \mathrm{g}$. Como se observa en la figura 1 en condiciones adecuadas de humedad y aireación la $\Sigma$ HAPs en los RBC se reduce en $65 \%$ en 120 días. Las tratamientos con muestras XI y XM logran una reducción del 93,4 y 95,6\%, respectivamente, mientras que con las muestras CI y CM logran una reducción del 81,3 y $85,5 \%$, respectivamente, esta situación puede deberse a que el material de compost XI y XM proviene de procesos parcialmente anaeróbicos y al someterse a un tratamiento aeróbico promueven los microorganismos que forman sustancias húmicas ${ }^{8}$. Estos microorganismos tienen la capacidad de degradar a los HAPs de forma similar como degradan la materia orgánica para producir sustancias húmicas ${ }^{3}$, por otro lado las muestras XI y XM, al tener mayor relación $\mathrm{C} / \mathrm{N}$, están en estado incompleto de maduración. Por otro lado, se observa que entre los 30 primeros días se logra una remoción mayor al $60 \%$ de la $\Sigma H A P s$ para todas las muestra de compost con RBC. En la muestra de solo RBC se logra el $60 \%$ de la $\Sigma$ HAPs recién a los 90 días.

Tabla 2. Contenido de HAPs en muestra de RBC

\begin{tabular}{cccccc}
\hline HAPs & FET & $\begin{array}{c}\text { Límite de } \\
\text { Detección }\end{array}$ & Unidades & $\begin{array}{c}\text { Extracto } \\
\text { de RBC } \\
(\mathrm{mg} / \mathrm{L})\end{array}$ & $\begin{array}{c}\text { RBC } \\
\mu \mathrm{g} / \mathrm{g}\end{array}$ \\
\hline NAP & 0,001 & 0,00007 & $\mathrm{mg} / \mathrm{L}$ & $\mathrm{ND}$ & $\mathrm{ND}$ \\
ACY & 0,001 & 0,00007 & $\mathrm{mg} / \mathrm{L}$ & $\mathrm{ND}$ & $\mathrm{ND}$ \\
ACE & 0,001 & 0,00007 & $\mathrm{mg} / \mathrm{L}$ & $\mathrm{ND}$ & $\mathrm{ND}$ \\
FLO & 0,001 & 0,00007 & $\mathrm{mg} / \mathrm{L}$ & $\mathrm{ND}$ & $\mathrm{ND}$ \\
PHE & 0,001 & 0,00007 & $\mathrm{mg} / \mathrm{L}$ & 0,0248 & 0,1243 \\
ANT & 0,01 & 0,00007 & $\mathrm{mg} / \mathrm{L}$ & $\mathrm{ND}$ & $\mathrm{ND}$ \\
FLA & 0,001 & 0,00007 & $\mathrm{mg} / \mathrm{L}$ & 0,0643 & 0,3216 \\
PYR & 0,001 & 0,00007 & $\mathrm{mg} / \mathrm{L}$ & 0,1108 & 0,5543 \\
BaA & 0,1 & 0,00007 & $\mathrm{mg} / \mathrm{L}$ & 0,0476 & 0,2381 \\
CHR & 0,01 & 0,00007 & $\mathrm{mg} / \mathrm{L}$ & 0,0483 & 0,2415 \\
BbF & 0,1 & 0,00007 & $\mathrm{mg} / \mathrm{L}$ & 0,0559 & 0,2795 \\
BkF & 0,1 & 0,00007 & $\mathrm{mg} / \mathrm{L}$ & 0,0261 & 0,1306 \\
BaP & 1,0 & 0,00007 & $\mathrm{mg} / \mathrm{L}$ & 0,0311 & 0,1558 \\
IcdP & 0,1 & 0,00007 & $\mathrm{mg} / \mathrm{L}$ & 0,0396 & 0,1980 \\
DahA & 1 & 0,00007 & $\mathrm{mg} / \mathrm{L}$ & 0,0151 & 0,0754 \\
BghiP & 0,01 & 0,00007 & $\mathrm{mg} / \mathrm{L}$ & 0,0808 & 0,4040 \\
LHAPs & & & & 0,5447 & 2,7234 \\
BaPeq & & & & 0,05648 & 0,2824 \\
\hline & & & & &
\end{tabular}


Naftaleno (NAP); Acenaftileno(ACY); Acenafteno (ACE); Fluoreno (FLO); Fenantreno (PHE); Antraceno (ANT); Fluoranteno (FLA); Pireno (PYR); Benz[a]antraceno (BaA); Criseno (CHR); Benzo[b]fluoranteno(BbF); Benzo[k]fluoranteno (BkF); Benzo[a]pireno (BaP); Indeno[1,2,3-cd]pireno (IcdP); Dibenzo[a,h]antraceno (DahA); Benzo[ghi]perileno (BghiP); Sumatoria de HAPs (इHAPs); Benzo[a]pireno equivalente (BaPeq)
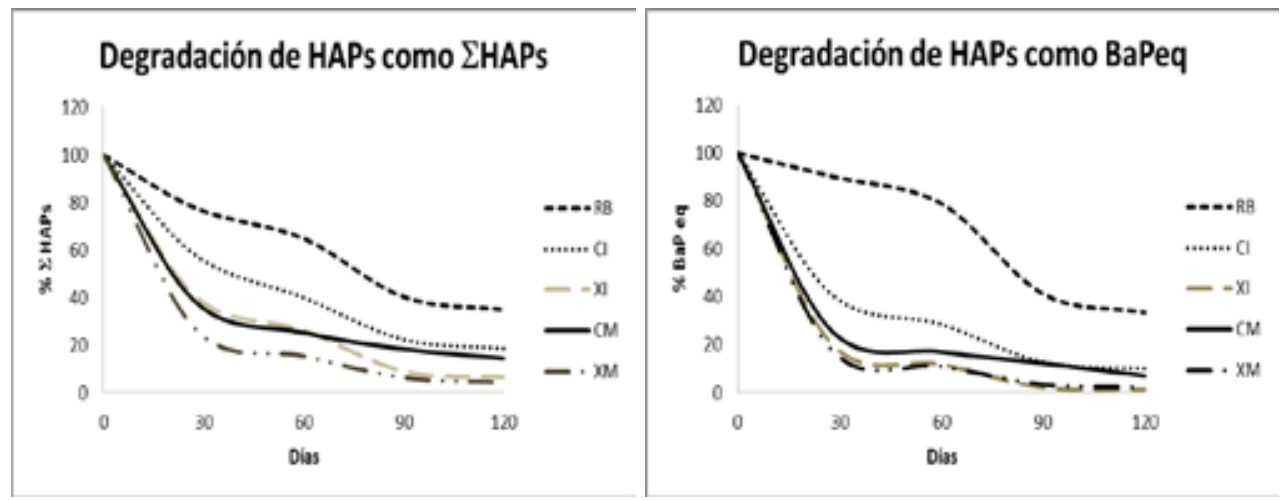

Figura 1. Degradación de HAPs como $\Sigma$ HAPs y BaPeq

Tabla 3. Tiempo de vida media de los diferentes HAPs en residuos de barrido de calles y tratamiento con compost

\begin{tabular}{lcccccccc}
\hline PAH & \multicolumn{9}{c}{$\mathrm{t}(1 / 2)$ días } & & & Anillo & $\begin{array}{c}\text { Promedio } \\
\text { grupo }\end{array}$ & $\begin{array}{c}\text { Literatura } \\
\text { suelos }^{10}\end{array}$ \\
\hline FTH & RRBB & CM & XM & CI & XI & & 28 & $110-4745$ \\
PYR & 88 & 10 & $<2$ & 12 & 16 & 4 & & 250 \\
BaA & 22 & 34 & 20 & 32 & 34 & 4 & & $240-730$ \\
CRY & 69 & 45 & 26 & 45 & 33 & 4 & & $87-5183$ \\
BbF & 83 & 49 & 18 & 41 & 46 & 5 & 34 & $139-4015$ \\
BaP & 50 & 4 & $<2$ & 10 & $<2$ & 5 & & $151-5329$ \\
DahA & 135 & 20 & $<2$ & 42 & 11 & 5 & & $240-730$ \\
BghiP & 181 & 96 & 44 & 110 & 46 & 6 & 75 & $173-657$ \\
IcdP & 134 & 35 & 10 & 63 & 27 & 6 & & $58-730$ \\
\hline
\end{tabular}

Como se observa en la tabla 3, sobre la biodegradabilidad de cada HAP, el tiempo de vida media promedio de los HAPs se incrementa con el número de anillos de los HAPs, No se ha detectado presencia de HAPs de 3 anillos debido a que tienen gran volatilidad y solubilidad relativa en agua cuando son biodegradados. Los HAPs de 4 anillos tienen un tiempo de vida media promedio de 28 días, estos compuestos son más hidrofóbicos, menos solubles en agua y tienen una gran afinidad con la materia orgánica del compost, lo que disminuye su disponibilidad, condición fundamental para su biodegradación. Los HAPs de 5 anillos tienen 
un tiempo de vida media promedio de 34 días superior al de 4 anillos, de estos se observa que el $\mathrm{BaP}$ tiene un menor tiempo de vida media, lo que sugiere una mayor biodegradación debido a su mayor solubilidad que los otros de 5 anillos. Los HAPs de 6 anillos menos solubles tienen un tiempo de vida media promedio de 75 días. La biodegradación de los HAPs sigue un mecanismo de mono o dioxidación en diferentes posiciones, seguido por una deshidratación de los dihidrodioles y una ruptura oxidativa usualmente en posición meta ${ }^{23-25}$.

Los tiempos de vida media de los HAPs en muestras de barrido de calles son menores que los presentados en la literatura de suelos y mayores que los presentes en las muestras de compost, debido a que la biodegradación mediada por hongos y bacterias depende de la capacidad de los hongos para adaptarse al sustrato (mayor en compost) y la naturaleza de los HAPs como fuente de carbono para la comunidad microbiana (mayor en residuos de barrido de calles y mezclas con compost que en los suelos) $)^{25}$.

\section{Efecto ecotóxico y tóxico de los lixiviados de las muestras tratadas y sin tratar}

El efecto tóxico, riesgo carcinogénico evaluado como el contenido de BaPeq, como se observa en la figura 1 , se reduce en más del $66 \%$ en todos los tratamientos con compost en 30 días, los tratamientos CI y CM presentan una menor remoción que XI y XM. La muestra de residuos de barrido de calles logra alcanzar una remoción del 60\% recién a los 90 días, requiere de una fase previa de acondicionamiento.

Como se observa en la figura 2 las muestras inmaduras XI y X después de un tiempo de 90 días de tratamiento incrementan su efecto ecotóxico sobre daphnias, en el caso de las muestras maduras CM y XM esto sucede a los 28 días. Este comportamiento puede deberse a la formación de productos intermediarios con mayor solubilidad en agua como epóxidos y dihidrodioles debido a procesos cometabólicos de los $\mathrm{HAPs}^{26}$ en la mezcla de residuo de barrido de calles y compost. Por otro lado las muestras maduras inician el proceso de degradación rápidamente debido a la presencia de organismos con capacidad de degradar ácidos húmicas en condiciones aeróbicas, en el caso de las muestras inmaduras es necesario un periodo de adaptación mayor de 60 días. 

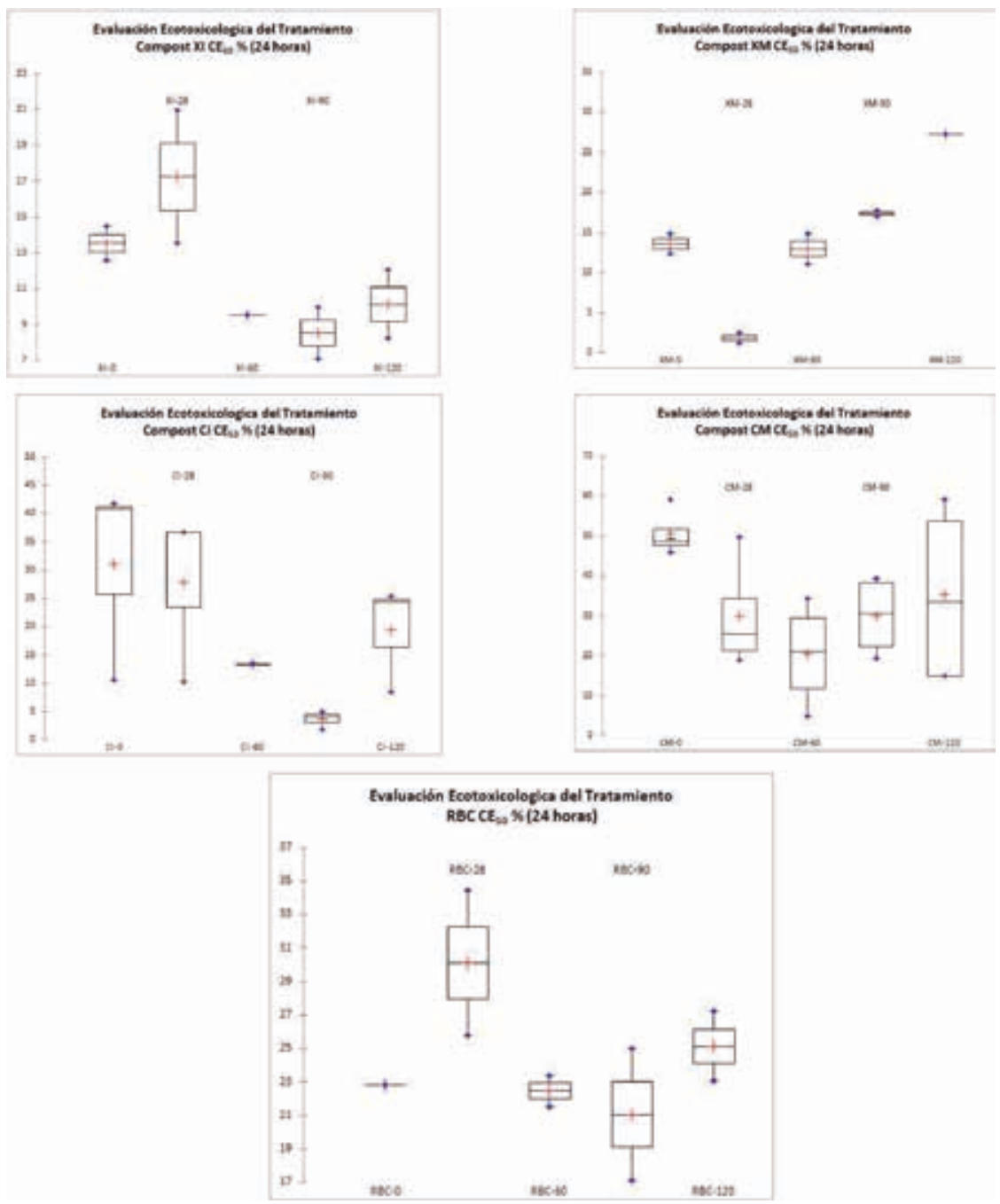

Figura 2. Efecto Ecotoxicológico del Tratamiento $\mathrm{CE}_{50} 24$ horas evaluado sobre Daphnia magna $(\mathrm{n}=4$ ensayos) 
Como se observa en la figura 3, el índice de germinación evaluado sobre Lactuca sativa presenta un compartimiento irregular, en el caso de $\mathrm{RBC}$ los valores de índice de germinación son menores a 2,0, los tratamientos con muestras XI y XM, disminuyen su índice de germinación a los 28 días similar a lo observado con las daphnias.

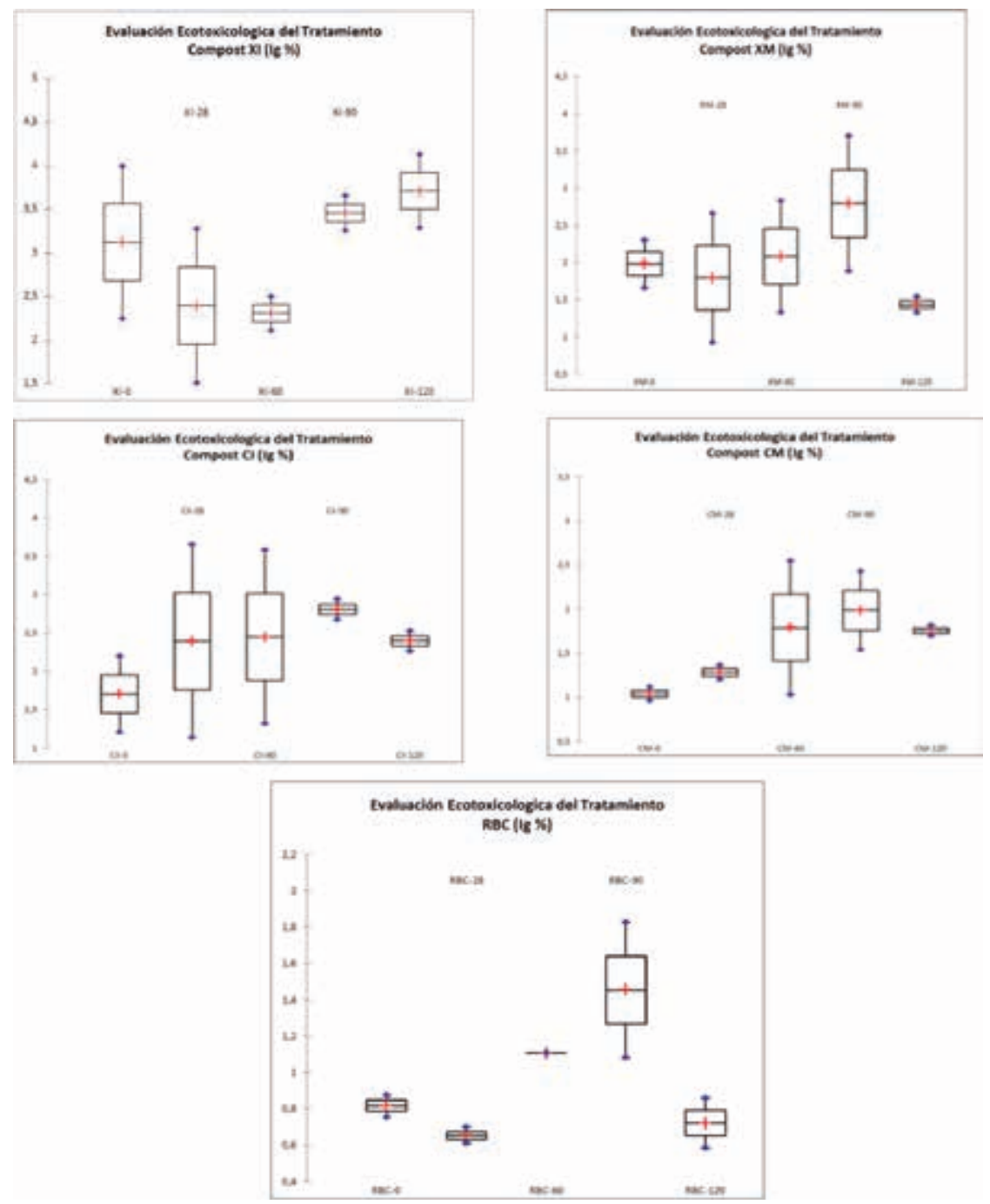

Figura 3. Efecto Ecotoxicológico del Tratamiento Ig (120 horas) evaluado sobre Lactuca sativa ( $\mathrm{n}=6$ ensayos) 
Las muestras CI y CM incrementan su índice de germinación hasta los 90 primeros días, a los 120 de tratamiento el índice de germinación disminuyen por motivos que requieren mayor estudio.

Los valores de ecotoxicidad de los tratamientos fueron convertidos en valores de unidad tóxica con la finalidad de poder tener todos los resultados en valores adimensionales que son aditivos y comparables. Los valores de las UT fueron comparados con el promedio de toxicidad y utilizando una escala arbitraria ${ }^{20,27}$, se clasificó la toxicidad de los residuos como sin toxicidad (st) $<1 \mathrm{UT}$, toxico (t) $1-100 \mathrm{UT}$, muy tóxico (mt) $11-100 \mathrm{UT}$, extremadamente tóxico (et) $>100$ UT. Como se observa en la tabla 4, los tratamientos con muestras de compost inmaduros, al cabo de 120 días, han formado metabolitos oxidados con mayor solubilidad y efecto tóxico, pueden ser compuestos epóxidos o dihidroxidos de HAPs ${ }^{26}$. En el caso de tratamientos con muestras de compost maduro CM y XM la ecotoxicidad de las muestras tratadas después de 120 días disminuye, probablemente debido a la formación de compuestos menos tóxicos comparados con los residuos de barrido de calles.

Tabla 4. Unidad tóxica de todos los ensayos ecotoxicológicos a 120 días de tratamiento

\begin{tabular}{cccccc}
\hline & \multicolumn{5}{c}{ Unidad Tóxica } \\
Residuo & Efecto & \\
\cline { 3 - 6 } & $24 h$ & $48 h$ & $\begin{array}{c}\% \\
\text { gaphnia magna }\end{array}$ & $\begin{array}{c}\text { Elongación } \\
\text { radicula }\end{array}$ & Ecotoxicidad \\
\cline { 3 - 6 } & 3,6753 & 4,3348 & 1,7826 & 6,5359 & $4,1(\mathrm{t})$ \\
\hline RB & 4,1054 & 11,8315 & 1,1896 & 3,4188 & $5,1(\mathrm{t})$ \\
CI & 8,3043 & 12,1271 & 1,0000 & 2,6954 & $6,0(\mathrm{t})$ \\
XI & 1,6893 & 1,9257 & 1,7082 & 3,3272 & $2,2(\mathrm{t})$ \\
CM & 3,6717 & 3,6724 & 1,8632 & 3,7523 & $3,2(\mathrm{t})$ \\
XM & & & & & \\
\hline
\end{tabular}

En la matriz de correlación de Pearson de la tabla 5, se observa que la humedad presenta correlación positiva con la pérdida por ignición (PPI) y relación $\mathrm{C} / \mathrm{N}$, presenta correlación negativa con el porcentaje de ácidos húmicos (HA) y contenido de $\Sigma$ HAPs al final del tratamiento, esto puede deberse a que las muestras húmedas provenientes de XRunner presentan mayor contenido de material orgánico inestable, los microorganismos al adaptarse a este medio cometabolizan a los HAPs en el proceso de formación de ácidos húmicos. La pérdida por ignición se correlaciona positivamente con la relación $\mathrm{C} / \mathrm{N}$, ecotoxicidad y negativamente con el contenido de HAPs, esto nos indica que las muestras con mayor contenido de carbono no estable tiene menor cantidad de ácidos húmicos, pero durante el tratamiento cometaboliza los HAPs en el proceso de producción de ácidos húmicos, generando metabolitos ecotóxicos de epóxidos o dihidroxidos de HAPs ${ }^{26}$. El porcentaje de ácidos húmicos se correlaciona positivamente con el \% $\%$ HAPs y \% BaPeq luego de 120 días de tratamiento, esto se debe a que las muestras con bajo contenido de ácidos húmicos 
provienen de XRunner, donde han sido tratados en condiciones parcialmente anaeróbicas y durante el tratamiento aeróbico han promovido la degradación de la materia orgánica en ácidos húmicos mediante microorganismos capaces de cometabolizar HAPs, reduciendo su concentración ${ }^{3,8,26}$.

Tabla 5. Correlación de parámetros toxicológicos, ecotoxicológicos, concentraciones de HAPs al final del tratamiento y composición del compost.

\begin{tabular}{|c|c|c|c|c|c|c|c|c|}
\hline Variables & $\%$ Humedad & Ceniza & PPI & $\mathrm{C} / \mathrm{N}$ & $\% \mathrm{HA}$ & Ecotoxicidad & $\% \Sigma$ HAPs & $\% \mathrm{BaPeq}$ \\
\hline$\%$ Humedad & 1 & $-0,983$ & 0,869 & 0,772 & $-0,745$ & 0,383 & $-0,857$ & $-0,948$ \\
\hline Ceniza & & 1 & $-0,932$ & $-0,821$ & 0,775 & $-0,530$ & 0,822 & 0,911 \\
\hline PPI & & & 1 & 0,675 & $-0,584$ & 0,786 & $-0,570$ & $-0,700$ \\
\hline $\mathrm{C} / \mathrm{N}$ & & & & 1 & $-0,990$ & 0,368 & $-0,897$ & $-0,855$ \\
\hline$\% \mathrm{HA}$ & & & & & 1 & $-0,232$ & 0,930 & 0,868 \\
\hline Ecotoxicidad & & & & & & 1 & $-0,041$ & $-0,152$ \\
\hline$\Sigma$ HAPs & & & & & & & 1 & 0,975 \\
\hline$\% \mathrm{BaP}$ eq & & & & & & & & 1 \\
\hline
\end{tabular}

\section{CONCLUSIONES}

Los HAPs pueden ser degradados utilizando tratamientos con compost, alcanzado porcentajes de remoción mayores al 60\% en 30 días y 80\% en 120 días, siguiendo rutas cometabólicas. Muestras de compost que provienen de un proceso anaeróbico antes del aeróbico tienen mayor capacidad para degradar HAPs, alcanzando valores de hasta el 90\% de remoción.

La degradación de los HAPs, en muestras de compost, forma inicialmente metabolitos más tóxicos que incrementan el efecto tóxico, principalmente entre los días 28 a 60, esto debido a que estos son más solubles en agua haciendo más biodisponibles, luego esta toxicidad disminuye por la biodegradación de los metabolitos y la disminución de la concentración de los HAPs.

Las muestras con compost maduro generan menores efectos ecotoxicológicos y toxicológicos.

\section{AGRADECIMIENTO}

Se agradece a la Dirección General de Investigación de la PUCP (DGI) que a través de los Proyectos anuales de investigación básica y aplicada 2013-0084 en cooperación con el Centro de Investigación en Química, Toxicología y Biotecnología Ambiental (CIQTOBIA) del Departamento Académico de Química de la UNALM financiaron la investigación. 


\section{REFERENCIAS BIBLIOGRÁFICAS}

1. Kästner M, Lotter S, Heerenklage J, Breuer-Jammali M, Stegmann R, Mahro B. Fate of 14C-labeled anthracene and hexadecane in compost-manured soil. Appl Microbiol Biotechnol. 1995;43(6):1128-35.

2. Kästner M, Mahro B. Microbial degradation of polycyclic aromatic hydrocarbons in soils affected by the organic matrix of compost.Appl Microbiol Biotechnol.1996;44(5):668-75.

3. Reid BJ, Fermor TR, Semple KT. Induction of PAH-catabolism in mushroom compost and its use in the biodegradation of soil-associated phenanthrene. Environ Pollut. 2002; 118(1):65-73.

4. Plaza C, Xing B, Fernández JM, Senesi N, Polo A. Binding of polycyclic aromatic hydrocarbons by humic acids formed during composting. Environ Pollut. 2009;157(1):257-63.

5. Carlstrom CJ, Tuovinen OH. Mineralization of phenanthrene and fluoranthene in yardwaste compost. Environ Pollut. 2003;124(1):81-91.

6. Lorenzi D, Entwistle JA, Cave M, Dean JR. Determination of polycyclic aromatic hydrocarbons in urban street dust: implications for human health. Chemosphere. 2011;83(7):970-7.

7. Bundesgesetzblatt Für Die Republik Österreich (BGB1. II) - Ausgegeben am 14. August 2001 - Nr. 292. pp $1756-1776$.

8. Binner E., Smidt E., Lechner P. Humic Acids - A Quality Criteria for Composts. Proceedings of the US Composting Councils 15th Annual Conference \& Trade Show. 21-23 enero 2007; Orlando, USA.

9. Antizar-Ladislao B, Lopez-Real J, Beck AJ. Laboratory studies of the remediation of polycyclic aromatic hydrocarbon contaminated soil by in-vessel composting. Wast Manag. 2005; 25: 281-289.

10. Kúsmierz M, Oleszczuk P, Kraska P, Pałys E, Andruszczak S. Persistence of polycyclic aromatic hydrocarbons (PAHs) in biochar-amended soil. Chemosphere. 2016; 146: 272 -279 .

11. Dong T, Lee B. Characteristics, toxicity, and source apportionment of polycylic aromatic hydrocarbons (PAHs) in road dust of Ulsan, Korea. Chemosphere. 2009; 74:1245-1253.

12. United States Environmental Protection Agency. Compendium Method TO-13A. Determination of Polycyclic Aromatic Hydrocarbons (PAHs) in Ambient Air Using Gas Chromatography/Mass Spectrometry (GC/MS). En Compendium of Methods for the Determination of Toxic Organic Compounds in Ambient Air. Second Edition. Cincinnati: EPA; 1999. p13A $24-25$.

13. United States Environmental Protection Agency. Method 1311: Toxicity Characteristic Leaching Procedure. En Test Methods for Evaluating Solid Waste, Physical/Chemical Methods. Washington DC: Government Printing Office; 1991.

14. Mantis I, Voutsa D, Samara C. Assessment of the environmental hazard from municipal and industrial wastewater treatment sludge by employing chemical and biological methods. Ecotoxicol Environ Saf. 2005; 62: 397-407.

15. United States Environmental Protection Agency. Method 8100: Polynuclear Aromatic 
Hydrocarbons. En Test Methods for Evaluating Solid Waste, Physical/Chemical Methods. Washington DC: Government Printing Office; 1986.

16. Wik A, Dave G. Acute toxicity of leachates of tire wear material to Daphnia magnaVariability and toxic components. Chemosphere. 2006; 64: 1777-1784.

17. Hamdi H, Manusadzianas L, Aoyama I, Jedidi N. Effects of anthracene, pyrene and benzo[a]pyrene spiking and sewage sludge compost amendment on soil ecotoxicity during a bioremediation process. Chemosphere. 2006; 65: 1153-1162.

18. Manzo S, De Nicola F, De Luca FP, Maisto G, Alfani A. Assessment of the effects of soil PAH accumulation by a battery of ecotoxicological tests. Chemosphere. 2008; 71 : 1937-1944.

19. Sprague JB, Ramsay BA. Lethal effects of mixed copper and zinc solutions for juvenile salmon. J Fish Res Bd Can. 1965; 22: 425-432.

20. Manusadzianas L, Balkelyte ' L, Sadauskas K, Blinova I, Põllumaa L, Kahru A. Ecotoxicological study of Lithuanian and Estonian wastewaters: selection of the biotests, and correspondence between toxicity and chemical-based indices. Aquat Toxicol. 2003; 63: $27-41$.

21. Nisbet C, LaGoy P. Toxic equivalency factors (TEFs) for polycyclic aromatic hydrocarbons (PAHs). Reg Toxicol Pharmacol. 1992; 16: 290-300.

22. Wang W, Huang M, Kang Y, Wang H, Leung OW, Cheung KC, et al. Polycyclic aromatic hydrocarbons (PAHs) in urban surface dust of Guangzhou, China: Status, sources and human health risk assessment. Sci Total Environ. 2011; 409: 4519-4527.

23. Fuchs G. Oxidation of organic compounds. En Biology of the Prokaryotes. Lengeler JW, Drews G, Schegel HG (edits). Stuttgart: Thieme; 1999. p 187-233.

24. Izquierdo AR. Biodegradación de HAPs durante la biorremediación aeróbica de suelos contaminados con hidrocarburos del petróleo. Análisis de poblaciones bacterianas genes funcionales. [Tesis Doctoral] Barcelona: Universidad de Barcelona; 2013.

25. Folwell BD, McGenity TJ, Whitby C. Biofilm and Planktonic Bacterial and Fungal Communities Transforming High-Molecular-Weight Polycyclic Aromatic Hydrocarbons. App Environ Microbiol. 2016; 82: 2288 -2299.

26. Cerniglia CE. Biodegradation of polycyclic aromatic hydrocarbons. Biodegradation. 1992; 3: 351-368.

27. Persoone G, Goyvaerts M, Janssen C, De Coen W, Vangheluwe M. Cost effective acute hazard monitoring of polluted waters and waste dumps with the aid of Tox kits, Final Report. Commission of European Communities. Contract ACE 89/BE 2/D3. 1993. p. 600 . 